\title{
Modeling urban dynamics through GIS-based cellular automata
}

\author{
M. Batty ${ }^{\mathrm{a}, *}$, Yichun Xie ${ }^{\mathrm{b}}$, Zhanli Sun ${ }^{\mathrm{c}}$ \\ ${ }^{a}$ Centre for Advanced Spatial Analysis (CASA), University College London, 1-19 Torrington Place, London \\ WCIE $6 B T, U K$ \\ ${ }^{\mathrm{b}}$ Center for Environmental Information Technology and Application (CEITA), Eastern Michigan University, \\ Ypsilanti, MI 48197, USA \\ 'State Key Laboratory for Resources and Environment Information Systems (LREIS), Institute of Geography, \\ Chinese Academy of Sciences, Building 917, Datun Road, Anwai, Beijing 100101, People's Republic of China
}

\begin{abstract}
In urban systems modeling, there are many elaborate dynamic models based on intricate decision processes whose simulation must be based on customized software if their space-time properties are to be explored effectively. In this paper we present a class of urban models whose dynamics are based on theories of development associated with cellular automata (CA), whose data is fine-grained, and whose simulation requires software which can handle an enormous array of spatial and temporal model outputs. We first introduce the generic problem of modeling within GIS, noting relevant CA models before outlining a generalized model based on Xie's (1996, A general model for cellular urban dynamics. Geographical Analysis, 28, 350-373) "dynamic urban evolutionary modeling" (DUEM) approach. We present ways in which land uses are structured through their life cycles, and ways in which existing urban activities spawn locations for new activities. We define various decision rules that embed distance and direction, density thresholds, and transition or mutation probabilities into the model's dynamics, and we then outline the software designed to generate effective urban simulations consistent with GIS data inputs, outputs and related functionality. Finally, we present a range of hypothetical urban simulations that illustrate the diversity of model types that can be handled within the framework as a prelude to more realistic applications which will be reported in later papers. (C) 1999 Published by Elsevier Science Ltd. All rights reserved.
\end{abstract}

Keywords: Urban dynamics; GIS; Cellular automata; Growth probabilities; Spatial evolution; Population density

* Corresponding author. Tel.: +44-171-391-1781; fax: +44-171-813-2843.

E-mail addresses: m.batty@ucl.ac.uk (M. Batty),gis_xie@online.emich.edu (Yichun Xie), sunzl@1reis. ac.cn (Zhanli Sun) 


\section{Modeling spatial and temporal processes within GIS}

Models of complex systems with geographic properties, such as cities and ecologies, usually involve spatial and temporal processes which are difficult to embed within proprietary GIS. Operational urban models are often built around the link between land use and transport, while models of the urban economy invariably simulate the linkages between different industrial sectors (Wegener, 1994). These models require spatial and sectoral interactions to be handled which cannot be easily adapted to the functionality of current GIS software. Moreover, during the last 10 years, the various generations of static urban models which are still those most widely used in urban policy making, are being challenged by more explicitly dynamic models (Allen, 1998). These are based on processes of urban development with time rather than space as their central organizing principle and this makes their embedding within GIS software even more difficult than the explicit representation of spatial interactions.

There is, however, a class of urban models developing rather rapidly which are, at first sight, strongly consistent with GIS. These are models based on the principles of cellular automata (CA) where temporal processes of change are represented through entirely local actions which take place in the immediate proximity of the various objects which comprise the system of interest. In spatial systems, the objects are usually defined as cells which can take on various states and which are influenced by what is happening in other cells in their immediate neighborhood. Although CA models need not be spatial per se or spatial in the two-dimensional sense either, most models appeal to the idea of representing the spatial system on a regular lattice such as a grid. Dynamic change is thus conceived as change in a cell being a function of the states of those cells which comprise its first nearest neighbors. The association between cells and grids and raster-based representation in GIS is immediate while the fact that the dynamics of such models are based on the simplest kinds of spatial interaction suggest that they might be implemented rather easily within proprietary GIS, especially those which emphasize raster-based representation (Wagner, 1997). Moreover, the development of models of landscape change based on CA-like principles (Itami, 1994) has drawn on GIS representations, particularly in terms of the way data can be combined through spatial layers. The increasing use of satellite data for monitoring urban change has also spurred the development of simple models of urbanization which can be represented in GIS (Clarke, Hoppen \& Gaydos, 1997; Yeh \& Li, 1998) and the way now looks open for the development of new GIS software which incorporates an explicit time dimension but without abandoning the kinds of functionality that characterize the most successful applications of GIS.

It is of course possible to write software modules with special functionality which can be interfaced with GIS in a variety of ways (Batty \& Xie, 1994a, b). Models can be loosely coupled to GIS through their inputs and outputs although such linkages, albeit useful, are largely based on pragmatic approaches to linking together software designed for quite different purposes. Stronger couplings such as those available as extensions to the popular ArcView package can be developed easily but in all these cases, the software that is ultimately produced inevitably and rightly 'defers' to the 
structure of the GIS. This often makes the development of well-adapted models and analytical methods with very different representational structures from spatial data difficult, if not impossible. In the models developed here, it is hard to develop the required flexibility within proprietary GIS and thus the approach we have taken is to develop software from scratch with limited GIS-like functionality but directly oriented to the analysis of spatial dynamics. There are similar examples based on adapting the STELLA systems dynamics software to deal with spatial development (Mikula, Mathian, Pumain \& Sanders, 1996) and there are now packages which enable models to be developed based on functions for conventional dynamic modeling and computing with raster data (Burrough, 1998). But in general, these packages do not provide an appropriate environment for dynamic urban models based on CA which is the focus here.

Before we deal with the particular requirements of the model and its software, it is worth summarizing, albeit briefly, the basis for dynamic urban modeling which is central to CA. In essence, CA models are based on very simple reaction-diffusion equations (Tamayo \& Hartman, 1989). The state of any cell depends upon some function which reacts to what is already in that cell as well as some function which relates the cell to what is happening in its immediate neighborhood - the diffusion component. In some senses, all dynamics are based on such a representation. For example, population growth processes involve reactions which are births, deaths and survivors from the existing stock with diffusion from that stock taking place through migration (Batty, 1971). Burrough (1998) refers to similar dynamic components in ecological models as dealing with establishment (births), persistence (survivorship, deaths) and dispersion (migration). What is particularly attractive about CA models for simulating urban systems is that local action in such models can give rise to global forms which evolve or emerge spontaneously with no hidden hand directing the evolution of the macrostructure. Cities are examples par excellence of this phenomenon and there has been much excitement recently in demonstrating that the geometry of urban form emerges as a consequence of local action using ideas from fractals (Batty \& Longley, 1994). In fact, there are classes of fractal model based on the aggregation and/or percolation of diffusing particles which produce patterns with remarkable similarities to cities and these can be easily generated using CA (Batty, 1991; Makse, Havlin \& Stanley, 1995; Makse, Andrade, Batty, Havlin \& Stanley, 1998).

As well as the coincidence of raster-based GIS with cellular dynamics and the logic of associating local action in cities with the global patterns that emerge, there is a third reason for building models which use cellular grids as their basis of spatial representation. It is well known that the geometric configuration of the spatial units used to represent spatial data can have a profound effect upon subsequent analysis and interpretation. Spatial systems which neutralize the effect of configuration remove any bias which is caused by convoluted or distorting geometries. Grid-based systems, for example, turn variables which are spatially extensive into their densityintensity equivalents and this immediately means that comparisons can be made between different parts of the same system as well as between different systems. Grid-based systems also neutralize the effect of administrative decisions over 
boundary definitions which in contemporary cities are often drawn for political purposes.

Most CA models to date have been developed for hypothetical applications (Couclelis, 1997) but this has been largely due to two constraints: first the strict CA principle that all action takes place in the immediate vicinity of the object/cell in question suggests that such models have mainly pedagogic value as a means for illustrating the consequences of local action on global patterns. Second, CA models have usually been developed in software which is not adapted to deal with extensive lattices such as those that characterize real urban systems of interest. Where CA-like models have been developed such as in the Marseilles region (Meaille \& Wald, 1990), Cincinnati (White \& Engelen, 1993), the Bay Area (Clarke et al., 1997), the Washington/Baltimore corridor (Clarke \& Gaydos, 1998), Guandong (Yeh \& Li, 1998), and in Guanzhou (Wu, 1998), these two constraints have been relaxed in that special purpose software has been written.

However, to date, there is no software available for urban CA models which has been developed for both hypothetical as well as real applications, with the possible exception of the AUGH software from the Venice group (Rinaldi, 1998). Because applications are of such complexity, explorations of their form under hypothetical conditions are necessary if the operation of their dynamics is to be thoroughly understood. In this paper, we will develop both an approach to modeling (dynamic urban evolutionary modeling, DUEM) as well as a suite of software which can accept a very wide range of hypothetical as well as real applications. Our software is designed to take spatial systems with up to nine million cells whose full display lies well beyond conventional screen technology. As the software is designed around temporal rather than spatial processes, CA models can be run indefinitely, or for as long as the user requires. These features clearly limit the use of conventional GIS software for the kinds of applications and models dealt with here.

In the rest of this paper, we first present the strategy for DUEM which seeks to demonstrate how urban simulations can be developed to great effect using powerful methods of visualization built around GIS (Densham, 1996). We then outline the formal structure of the model in terms of the way a typical activity is generated and located in the cellular space. We have adapted the model to several land uses which provides a basis for the software we have written. We have detailed this software with an emphasis on how it provides an exploratory simulator for urban dynamics, and we have illustrated very different kinds of urban application, i.e. different kinds of city, which can be developed within the software. These hypothetical applications provide some insights into the range of possibilities that the simulator can embrace and act as a focus for the various empirical applications that we are currently developing.

\section{A formulation of the generic model}

Activities in urban systems follow life cycles which apply both to the physical stock that gets developed as well as the activities or uses that occupy the stock at 
different locations. Forrester (1969) was one of the first to distinguish between activities in terms of their life cycle in his Urban Dynamics model where he defined, new, mature and declining housing, industry and commercial land uses, each subject to different rates of growth (or decline) and different rates by which existing activities attracted or detracted from new growth in related locations. In DUEM, we make a distinction between initiating, mature and declining activities which at any point in time $t$ compose the stock of that activity which we call $A$. Dealing first with a single activity which we treat generically, the total activity at time $t, A(t)$, is defined as:

$$
A(t)=A^{\mathrm{i}}(t)+A^{\mathrm{m}}(t)+A^{\mathrm{d}}(t)
$$

where $A^{\mathrm{i}}(t)$ is the total activity treated as initiating, $A^{\mathrm{m}}(t)$ is the total treated as mature, and $A^{\mathrm{d}}(t)$ is the total that is declining and which will disappear from the stock or 'die' in the next time period $t+1$. Most initiating activity is not new to that time period $t$ but is composed of all that activity which is able to generate new growth in contrast to mature activity, which no longer generates growth. New growth in the time period $t$ is already added into initiating activity $A^{\mathrm{i}}(t)$.

The way activity moves through its life cycle depends upon how it is converted and for this we need to know its location $j$. The process of deciding whether an activity $A^{j}(t)$ is in an initiating or mature state depends upon when it was first established as new at time $t=\tau^{j}$. Then the probability of the activity being in the initiating state $\rho_{j}^{\mathrm{i}}(t)$ declines with time according to a typical decay function:

$$
\rho_{j}^{\mathrm{i}}(t)=\Lambda \exp \left\{-\lambda\left(\mathrm{t}-\tau_{j}\right)\right\}
$$

$\Lambda$ is a constant of proportionality which normalizes the distribution and $\lambda$ is a parameter reflecting the rate of decay from initiating to mature activity. This provides us with a first guess at the probability of the activity being mature $\Pi_{j}^{\mathrm{m}}(t)$ as:

$$
\Pi_{j}^{\mathrm{m}}(t)=1-\rho_{j}^{\mathrm{i}}(t)
$$

but from this, we must deduct the proportion $\rho_{j}^{\mathrm{d}}(t)$ which 'dies' in the next time period $t+1$. This is based on a further decay process which generates the final probability of the activity being mature $\rho_{j}^{\mathrm{m}}(t)$ as:

$$
\rho_{j}^{\mathrm{m}}(t)=\Pi_{j}^{\mathrm{m}}(t) \mathrm{M} \exp \left\{-\mu\left(t-\tau_{j}\right)\right\} .
$$

$\mathrm{M}$ is the relevant normalizing constant and $\mu$ is a decay parameter. The probability that the activity 'dies' in the next time period is:

$$
\rho_{j}^{\mathrm{d}}(t)=\Pi_{j}^{\mathrm{m}}(t)-\rho_{j}^{\mathrm{m}}(t)
$$

and from Eqs. (2) to (5) it is clear that these probabilities sum to 1, that is: 


$$
\rho_{j}^{\mathrm{i}}(t)+\rho_{j}^{\mathrm{m}}(t)+\rho_{j}^{\mathrm{d}}(t)=1 .
$$

In each time period, the actual status of the activity is determined by drawing a random number between 0 and 1 . If the number is less than $\rho_{j}^{\mathrm{i}}(t)$, activity is classed as initiating, if between $\rho_{j}^{\mathrm{i}}(t)$ and $\rho_{j}^{\mathrm{m}}(t)$ it is mature, and if above $\rho_{j}^{\mathrm{m}}(t)$, it is declining, thus disappearing from the stock during the next time interval.

The location of activity in the system can also be treated generically before we adapt it to various land use types. We consider the urban system is best represented at three spatial scales or levels: the level of the neighborhood $\Omega_{1}$, the field $\Omega_{2}$, and the region $\Omega_{3}$, with these three levels being nested within one another. In current versions of the model, this is a strict nesting although it can be relaxed if necessary. Each activity $A$ has the potential to influence growth and decline of the same or other activities in its neighborhood and in its field. The neighborhood is specified by a 'radius of affected area' parameter $r$ while the field that lies beyond this is measured by a 'free length of development' parameter. For example, if the neighborhood is specified as having a radius of 2 , then this means that the activity in question is located at the center of a grid of dimension $5 \times 5$ or $(2+1+2) \times(2+1+2)$, i.e. two steps beyond the central cell in question each way. A radius parameter of 1 means that the cell is in a neighborhood of $3 \times 3$ or $(1+1+1) \times(1+1+1)$-the so-called Moore neighborhood which is one of the most commonly used in CA modeling (Toffoli \& Margolus, 1987) - while if $r=0$, then there is no effective neighborhood.

The 'free length' parameter defines the extent of the field beyond the neighborhood within which it is contained. If this parameter is 5 say, with a neighborhood radius parameter of say 2 , then this means that the $5 \times 5$ neighborhood sits at the center of a field which is $15 \times 15$ or $(5+2+1+2+5) \times(5+2+1+2+5)$ in size. Various rules about development and redevelopment apply to the neighborhood and field. These are specified for each activity according to the following general principles. First, development is located in the neighborhood/field with respect to distance from the activity in the cell in question, and there are various parameters which control the way distance affects the decision to locate at any cell within the field. In essence, distance from existing development acts a deterrent to new development. Second, development occurs in a certain direction from the activity in question, thus reflecting how certain cities might grow in terms of sectors or wedges. There is complete control over direction through various parameters that can be set. Third, there are various constraints on development in the neighborhood of any potential cell for development in the field which are associated with the density of the same or different land uses in the neighborhood in question. Finally, there are transitions specified as probabilities of new activity generated from one type of land use occurring as another type of land use in the field, and these are defined by levels of density that must be achieved if certain types of land use are to be located. We will deal with these rules in turn.

Around each initiating activity $A_{j}^{\mathrm{i}}(t)$, we compute the probability that new activity $A_{k}(t)$ is to be located in the field at location $k$ according to the distance from the activity at location $j$. The type of activity is unimportant at this stage but the 
function used to compute this probability is. Before the simulation begins, we can select one of four functions - an inverse linear function of distance, negative exponential, inverse power, or a gamma-like function which combines the exponential and power (Xie, 1994, 1996). Here for illustrative purposes, we choose the negative exponential, and thus the probability $\Pi_{j k}(t)$ that a cell $k$ is chosen in the field centered on $j$ is given as:

$$
\Pi_{j k}(t)=\mathrm{Q} \exp \left\{-\alpha r_{j k}\right\}
$$

$\mathrm{Q}$ is scaling constant and $\alpha$ is a parameter controlling the decay effect of distance, $r_{j k}$, measured between locations $j$ and $k$. A second probability is also chosen and this is based on a distortion of the field in a predetermined direction. The user has the option of transforming the symmetric field around the activity $A_{j}^{\mathrm{i}}(t)$ in a variety of ways. In each of the eight compass directions around the point $j$, it is possible to exaggerate the weight of any of these. If each of these compass directions is given an equal weight, then it possible to gradually suppress the weight of the NW, NE, SE, and SW directions or to give increasing weight to any combination of these directions. This function is fairly elaborate and a demonstration of its use is postponed until the model is demonstrated later. If each of the eight directions is given an angular heading $\theta_{z}, z=1,2, \ldots, 8$ and a weight $\omega_{z}$, then the probability of growth taking place in any cell in the direction $x$, is given as:

$$
\rho_{x \mid j}=\omega_{z} \theta_{z} / \Sigma_{z} \omega_{z} \theta_{z}
$$

The combined probability $\rho_{j k}(t)$ of growth taking place in any cell $k$ in the field is then computed by combining Eqs. (6) and (7) as:

$$
\rho_{j k}(t)=f\left\{\Pi_{j k}(t), \rho_{x \mid j}\right\}
$$

A random number between 0 and 1 is drawn to determine the particular cell $\kappa(=\mathrm{k})$ which remains in the development pool at this stage.

Whether or not this cell is developed and what activity is actually located there depends upon three other functions. First, the cell in question $\kappa$ is examined with respect to its classification in the wider region $\Omega_{3}$. If it is classed as developable, it survives but if not, due to exogenous physical constraints, it is ruled out from further consideration. Second, if the cell is one which was originally developed but is now vacant, then there may be a moratorium on its development for this is one of the parameters that is set up prior to the simulation when the life cycle of the activity is examined. If it survives this test, then a third test involving the density of other activities in the neighborhood of the chosen cell $\kappa$ is invoked. Let us consider one land use only although this test can be extended to any number. The density of this land use in the neighborhood of $\kappa$ is computed as $\delta$ and if this density is greater (or less) than a certain threshold $\sigma$ depending upon the activity in question, then the cell becomes 'actually' developable. It now becomes necessary to determine what activity is developed. 
It may seem strange to consider growth generated from one type of activity being a different type but this simply reflects the linkages between activities. For any pair of activities $\chi$ and $\phi$, say, then there is a probability that one activity $\chi$ gives rise to another activity $\phi$ and these probabilities are defined as $\rho_{\chi \phi}$. The matrix of probabilities is a transition matrix, asymmetric in form, which is used to determine what particular activity is generated in cell $\kappa$. These transition probabilities are determined in advance and in the current model are not time-dependent. Moreover, the matrix is strongly diagonal in that the greatest probabilities are those which relate to one activity spawning the same, that is $\left(\rho_{\chi \chi}, \rho_{\phi \phi}\right)>>\left(\rho_{\chi \phi}, \rho_{\phi \chi}\right)$. These probabilities are determined by the user prior to the simulation but they depend upon the density of different activities in the neighborhood around the cell $\kappa$. This cell will always be allocated some activity but the particular activity will depend upon the composition of uses in the neighborhood. If the neighborhood only has a single use, then the probability that the activity will be that use is always higher than in neighborhoods of mixed use which lead to more heterogeneity in the chosen activities.

What we do not have in the model at the present time are transitions from one land use to another based on mutations which reflect the possibility that one land use, either in its initiating or mature cycle, can mutate to another without dying first and the plot being redeveloped. This kind of mutation happens in urban systems and although we are introducing this effect in a newer version of DUEM, all the experiments reported here are based on development without mutation. At this stage, it is worth summarizing how development emerges from this model. All new development is initiated in fields centered on $\left\{A_{j}^{\mathrm{i}}(t)\right\}$. Development which is mature does not initiate new development but remains passive in the relevant time period, although, due to the nature of the life cycle there is the possibility, at any time, of activity which has been initiating or mature in the previous period, becoming mature or initiating in the next. Activity which is declining at the start of the time period $\left\{A_{j}^{\mathrm{d}}(t)\right\}$ has disappeared from the system by $t+1$, while new activities $\left\{A_{k}^{\mathrm{n}}(t)\right\}$ originating in the developable cells $\kappa$ which survive all the various tests, are added to the stock.

Various stock and flow equations which govern the development process through time, can now be stated. As each cell $j$ can only ever contain one activity, then equations which are specific to locations are trivial. Thus stocks and flows need only be stated for the entire system which we represent as summations across the set of locations $\{j\}$. Then:

$$
A(t+1)=\Sigma_{j} A_{j}^{\mathrm{n}}(t)+\Sigma_{j} A_{j}^{\mathrm{i}}(t)+\Sigma_{j} A_{j}^{\mathrm{m}}(t)-\Sigma_{j} A_{j}^{\mathrm{d}}(t)
$$

is a typical birth $\left\{\Sigma_{j} A_{j}^{\mathrm{n}}(t)\right\}$, death $\left\{\Sigma_{j} A_{j}^{\mathrm{d}}(t)\right\}$ and survivorship $\left\{\Sigma_{j} A_{j}^{\mathrm{i}}(t)+\Sigma_{j} A_{j}^{\mathrm{m}}(t)\right\}$ equation with the analogous discrete change flow equation computed as:

$$
\Delta A(t+1)=A(t+1)-A(t)=\Sigma_{j} A_{j}^{\mathrm{n}}(t)-\Sigma_{j} A_{j}^{\mathrm{d}}(t) .
$$

Total changes in activity are not yet constrained to reflect predetermined growth rates. In fact the entire growth (or decline) profile of the system is determined by the 
way in which cells are selected. Clearly the number of seeds and their configuration, one to another, has some effect on the growth generated but it is not possible in advance to determine how much growth or decline there will be. However, at the end of each time period, as Eqs. (9) and (10) indicate, it is possible to compute the growth rate $\Gamma(t+1)$ for the system (and its parts if necessary) as:

$$
\Gamma(t+1)=A(t+1) / A(t)=\{1+\Delta A(t+1) / A(t)\}
$$

From Eq. (11), it is possible to reset various parameters in the system to reflect requirements for increased or decreased growth. Another important rate is total initiating activity to total activity which is a measure of how the system is performing in growth terms. This rate $\gamma(t+1)$ can be defined as:

$$
\gamma(t+1)=\Sigma_{j} A_{j}^{\mathrm{i}}(t) / A(t+1)
$$

We will not attempt a detailed analysis of growth paths in this paper, but in developing the framework of DUEM for empirical applications, the first of which is to the town of Ann Arbor, MI, we will extend the model to handle such issues in a completely transparent way. In the current paper, our concern is with introducing the software and showing the range of hypothetical applications that are possible with DUEM.

\section{Adapting the model to distinct land uses}

The model becomes considerably more complex when different activities are considered. Each activity invariably has a different rule set associated with development which is specified through various parameters. In the current version, we have translated activities into five distinct land uses: housing or residential population which we will define as P; resource and manufacturing employment or industry, E; service or commercial-retail-shopping activity, S; streets or transport routes, R; and vacant land, V. Although we have labeled these variables as if total population, employment etc. can be derived from them, the current model like all CA only deals with the physical allocation of land use, one unit of land use per cell. To compute total activities, cells must be aggregated across locations $\{j\}$ and this implies that for realistic applications, the level of resolution of the spatial system must be very fine. The model can of course be run for a subset of these land uses which minimally might contain a single land use and vacant land. It is thus possible to simulate urban development per se where the emphasis is on the transition from rural (vacant) land to urban although DUEM is designed for more elaborate applications where simulating the development of a series of interdependent activity/land use types is the focus.

We will describe the various rules used to define the dynamics of change associated with these five land uses before we provide an overview of how the model can be run. In locating four of these land uses-housing, industry, commerce, and 
transport routes/streets, the effect of the initiating activity for each of these uses on the location of new development in their respective fields operates in the same way with respect to the distance decay and directional effects. We dealt with this briefly in the previous section but here we must be a little more explicit concerning the possible options. The distance probability $\Pi_{j k}(t)$ given in Eq. (6) for the exponential form, can also be based on a simple linear inverse distance defined as $\Pi_{j k}(t)=Q-\alpha r_{j k}$, on an inverse power function of distance $\Pi_{j k}(t)=Q r_{j k}^{-\alpha}$, or on the modified gamma function $\Pi_{j k}(t)=Q r_{j k}^{-\alpha} \exp \left\{-\beta r_{j k}\right\}$ where the parameters must be specified in advance. In choosing these, the user must be careful to ensure that these are consistent with the dimensions of the spatial system; this should be possible for a wide range of applications because the DUEM software enables levels of spatial resolution up to $3000 \times 3000$ to be handled.

The direction in which allocation takes place within the field $\Omega_{2}$ is determined by a graphical interface in which the user adjusts the weight of each sector in the eight compass directions. We illustrate this procedure in Fig. 1 where we show two examples of varying the weights of the compass directions with the weights proportional to the areas of the sectors shown. The user can opt for standard weights by systematically reducing the effect of the NE, NW, SE and SW directions from a standard level in which all eight direction are weighted equally, using the left, middle, and right sequence buttons in the dialog. As Fig. 1 also shows, the user can then vary these weights substantially by clicking on one of the eight directions and skewing its effect across a range from 0 to 100 using the slider. Somewhat more important is the way the distance and direction probabilities are combined for each land use. Although the distance model is able to compute a probability for each cell
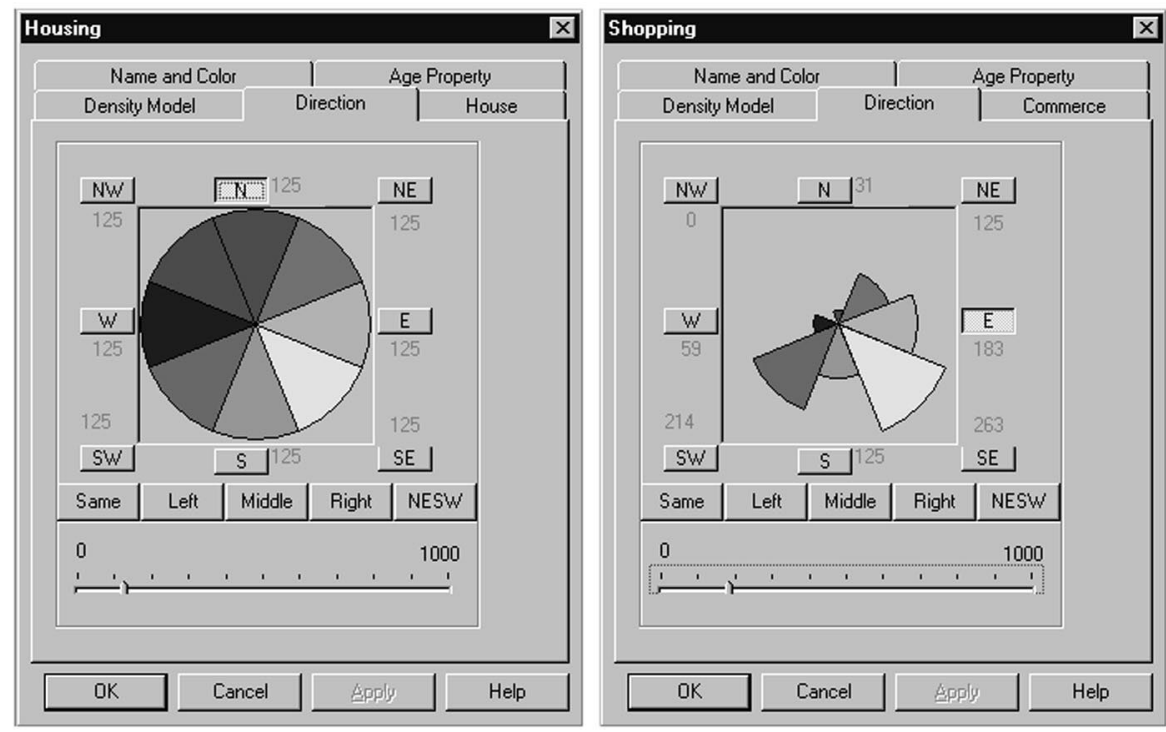

Fig. 1. Specifying the directional weights. 
$k$ around the initiating activity $j$, we aggregate the cell probabilities by distance bands around $k$. Using the example in Eq. (6),

$$
\Pi_{j \mathrm{~B}}(t)=\Sigma_{k \subset \Omega \mathrm{B}} \Pi_{j k}(t)
$$

where $\Omega_{\mathrm{B}}$ is the relevant band in which the appropriate cells $k$ are located. The actual band chosen is then decided randomly from the set of probabilities $\left\{\Pi_{j \mathrm{~B}}(t)\right\}$. The probability $\rho_{x \mid j}$ where $x$ is the direction from $j$, is then used to select the actual direction $X$. As the distance bands are only one cell wide, the intersection of the direction set $\Omega_{\mathrm{X}}$ and the band set $\Omega_{\mathrm{B}}$, that is $\Omega_{\mathrm{X}} \cap \Omega_{\mathrm{B}}$, determines the cell $\kappa$ in question.

The rules for further selection are more specific in that each individual land use has particular characteristics, which imply that the rules are always defined in relation to the land use of the initiating activity. We will deal with residential activity $\mathrm{P}$ first as this is the most extensive, occupying up to one half of all land classified as urban. The cell $\kappa$ is only acceptable for development initiated from housing if the density of street nodes - that is key vertices in the transport network between which routes exist - exceeds a certain threshold. This is to ensure that the land use to be developed is adjacent to streets in its neighborhood which will service and physically connect the relevant cell to the rest of the transportation system. This clearly depends upon the size of the neighborhood and the density of street nodes that define the threshold but in general, the user needs to be careful in defining reasonable values to ensure that a connected city develops.

Once a cell has passed this test, and assuming that it is compatible with any constraints on development posed by the region $\Omega_{3}$, the land use type can be selected. Development initiated by housing can be any one from the three main types: housing, industry and commerce but there are two different sets of transition probabilities that determine this. First, if all the activity in the neighborhood around $\kappa$ is housing, then the probabilities of the development at $\kappa$ for housing, industry or commerce are given as $\rho_{\mathrm{PP}}, \rho_{\mathrm{PE}}$ and $\rho_{\mathrm{PS}}$. If the land uses in the neighborhood of cell $\kappa$ are in mixed use, then a different set of probabilities apply and these are defined as $\pi_{\mathrm{PP}}, \pi_{\mathrm{PE}}$ and $\pi_{\mathrm{PS}}$. In general, we would expect $\rho_{\mathrm{PP}}>\pi_{\mathrm{PP}}$, and $\rho_{\mathrm{PE}}<\pi_{\mathrm{PE}}, \rho_{\mathrm{PS}}<\pi_{\mathrm{PS}}$ because housing which initiates development in a neighborhood which is predominantly residential has a higher probability of being housing in comparison to any other new activity in a mixed use neighborhood. This is one of the many areas of the model where it is important to specify rules for development that are logically plausible. At the same time it is clear that such rules, when considered in entirety, have a profound effect on the total activity which is generated.

Industry at $j$ which initiates new development, follows the same distance and directional rules used for housing. There is a similar constraint to that for housing on the density of street nodes that is invoked if the cell $\kappa$ is to be considered as developable. Compatibility with any regional constraints in $\Omega_{3}$ must also be observed. However, unlike activity which is initiated from housing, activity initiated from industry can only be industry. In this way we exercise some control over the totals being generated in that the ultimate total will depend upon that which is given at the start of the simulation. To make sure that the composition of the neighborhood around $\kappa$ is such 
that it is suitable for industry, a further constraint must be met: the proportion of cells in the neighborhood given over to housing and commerce must be less than a certain threshold if the cell is to be developed as industry. Of course, it would be entirely possible to use similar transitions to other land uses as those associated with the housing initiator but for the moment in this version of the model, such complexity has not been added. The commercial/retail land use sector follows the same logic as that used to initiate new development from industry. Commercial activity only initiates commercial, a street threshold exists to enable connectivity to be maintained, and the mix of housing and industry in the neighborhood of $\kappa$ must be less than a certain threshold for the cell at $\kappa$ to be developed as commercial activity.

Finally, we must examine the transport routes which are also created by the simulation. Such routes are specified as nodes from which new nodes are spawned and directly connected by street lengths. Nodes spawn nodes and no mutation to other land uses takes place. As streets follow urban development and urban development follows streets - as reflected in the circularity and positive feedback which urban change engenders - it is logical to suppose that the same inverse distance and directional rules as those used for housing, industry and commerce apply. To this, we must add constraints on minimum development in the neighborhood of any node spawned. We have set these up so that if (different) minimum proportions of housing, industry, or commerce occur in the neighborhood of the new node, then the new node is established. However, the street which is established when this node is joined up to the initiating node must be at least a given number of cells away from any existing streets in the field. This avoids duplication of routes, and in particular large groups of adjacent cells devoted entirely to transport use. In fact this constraint is important because it also establishes a bound on the density of transport routes. One problem of treating transport routes and nodes as cells of the same size as other land uses, is that in reality, transport routes are at a much finer scale than land parcels which marks the difference in urban geometry between line and area features; this constraint helps establish this.

We are now in a position to tie together the various land uses in the model and to compute total growth or decline which lies at the basis of exploring the extent to which realistic totals are generated. We have four substantive land uses that any time $t$, can be defined as $A_{j}^{\mathrm{P}}(t), A_{j}^{\mathrm{E}}(t), A_{j}^{\mathrm{S}}(t)$, and $A_{j}^{\mathrm{R}}(t)$. We define initiating and mature versions of these same land uses which are relevant to the time period just completed. Those which are initiating, are defined as $A_{j}^{\mathrm{Pi}}(t), A_{j}^{\mathrm{Ei}}(t), A_{j}^{\mathrm{Si}}(t)$, and $A_{j}^{\mathrm{Ri}}(t)$ while we also have vacant land use $A_{j}^{\mathrm{V}}(t)$ at time $t$. Stock equations can be stated for each of these land uses

$$
\begin{aligned}
& A^{\mathrm{P}}(t)=\Sigma_{j} A_{j}^{\mathrm{P}}(t), \text { and } A^{\mathrm{Pi}}(t)=\Sigma_{j} A_{j}^{\mathrm{Pi}}(t) \\
& A^{\mathrm{E}}(t)=\Sigma_{j} A_{j}^{\mathrm{E}}(t), \text { and } A^{\mathrm{Ei}}(t)=\Sigma_{j} A_{j}^{\mathrm{Ei}}(t) \\
& A^{\mathrm{S}}(t)=\Sigma_{j} A_{j}^{\mathrm{S}}(t), \text { and } A^{\mathrm{Si}}(t)=\Sigma_{j} A_{j}^{\mathrm{Si}}(t) \\
& A^{\mathrm{R}}(t)=\Sigma_{j} A_{j}^{\mathrm{R}}(t), \text { and } A^{\mathrm{Ri}}(t)=\Sigma_{j} A_{j}^{\mathrm{Ri}}(t)
\end{aligned}
$$

and totals are defined as: 


$$
A(t)=\Sigma_{\mathrm{q}} A^{\mathrm{q}}(t) \text { and } A^{\mathrm{i}}(t)=\Sigma_{\mathrm{q}} A^{\mathrm{qi}}(t)
$$

where the summation over $\mathrm{q}$ is over the set of four land uses $\{\mathrm{q}=\mathrm{P}, \mathrm{E}, \mathrm{S}, \mathrm{R}\}$. As in Eq. (10), we can compute growth rates $\Gamma^{\mathrm{q}}(t+1)$ for each of these land uses as well as the rates of active to total growth $\gamma^{\mathrm{q}}(t+1)$, both of which are important to the long term dynamics of the model. The growth rates for each land use are defined as:

$$
\begin{array}{ll}
\Gamma^{\mathrm{P}}(t+1)=A^{\mathrm{P}}(t+1) / A^{\mathrm{P}}(t), & \Gamma^{\mathrm{E}}(t+1)=A^{\mathrm{E}}(t+1) / A^{\mathrm{E}}(t), \\
\Gamma^{\mathrm{S}}(t+1)=A^{\mathrm{S}}(t+1) / A^{\mathrm{S}}(t), & \Gamma^{\mathrm{R}}(t+1)=A^{\mathrm{R}}(t+1) / A^{\mathrm{R}}(t),
\end{array}
$$

with the rates of initiating activity as:

$$
\begin{array}{ll}
\gamma^{\mathrm{P}}(t+1)=A^{\mathrm{Pi}}(t+1) / A^{\mathrm{P}}(t+1), & \gamma^{\mathrm{E}}(t+1)=A^{\mathrm{Ei}}(t+1) / A^{\mathrm{E}}(t+1), \\
\gamma^{\mathrm{S}}(t+1)=A^{\mathrm{Si}}(t+1) / A^{\mathrm{S}}(t+1), & \gamma^{\mathrm{R}}(t+1)=A^{\mathrm{Ri}}(t+1) A^{\mathrm{R}}(t+1) .
\end{array}
$$

These rates are computed by the software and provide essential diagnostics in determining how the model is performing. To progress the discussion further, it is now important to provide a brief outline of the software and the kinds of simulation that are currently being attempted within this framework.

\section{Exploring the model through its software}

The model software is written in Visual $\mathrm{C}++$ and runs on a PC under Windows 95/NT and later generations of operating system. In essence, we have created a selfcontained package through which the user can develop both hypothetical and real applications. The package also enables a very wide range of CA-like model types to be defined. A typical interface which is on-screen when the model is running is shown in Fig. 2 for an example where several land uses are located randomly at the beginning of the simulation. We will continually refer to this in the following discussion when we examine the various menu items and parameter options that the user must invoke to develop any application, but we will also use this example as part of the more substantive discussion of the model's properties which we begin in a later section. In essence, the interface consists of a series of pull-down menus from which various options can be selected with some of these options also displayed in tool bars as icons which enable the user to speed up selection.

The screen shot also shows that when the model is running, there are three window types displayed. As the model simulates urban development through time, two of these windows deal with spatial and temporal dynamics: the main window shows the pattern of urban development at time $t$ in terms of the five land uses and their locations, that is the set $\left\{A_{j}^{\mathrm{P}}(t), A_{j}^{\mathrm{E}}(t), A_{j}^{\mathrm{S}}(t), A_{j}^{\mathrm{R}}(t)\right.$, and $\left.A_{j}^{\mathrm{V}}(t)\right\}$, and is updated throughout the simulation. The second window type contains four graphs showing the trajectories of various activity totals, namely $A^{\mathrm{P}}(t), A^{\mathrm{E}}(t), A^{\mathrm{S}}(t)$ and $A(t)$ as well as the initiating totals $A^{\mathrm{Pi}}(t), A^{\mathrm{Ei}}(t)$, and $A^{\mathrm{Si}}(t)$. These graphs of course are updated 


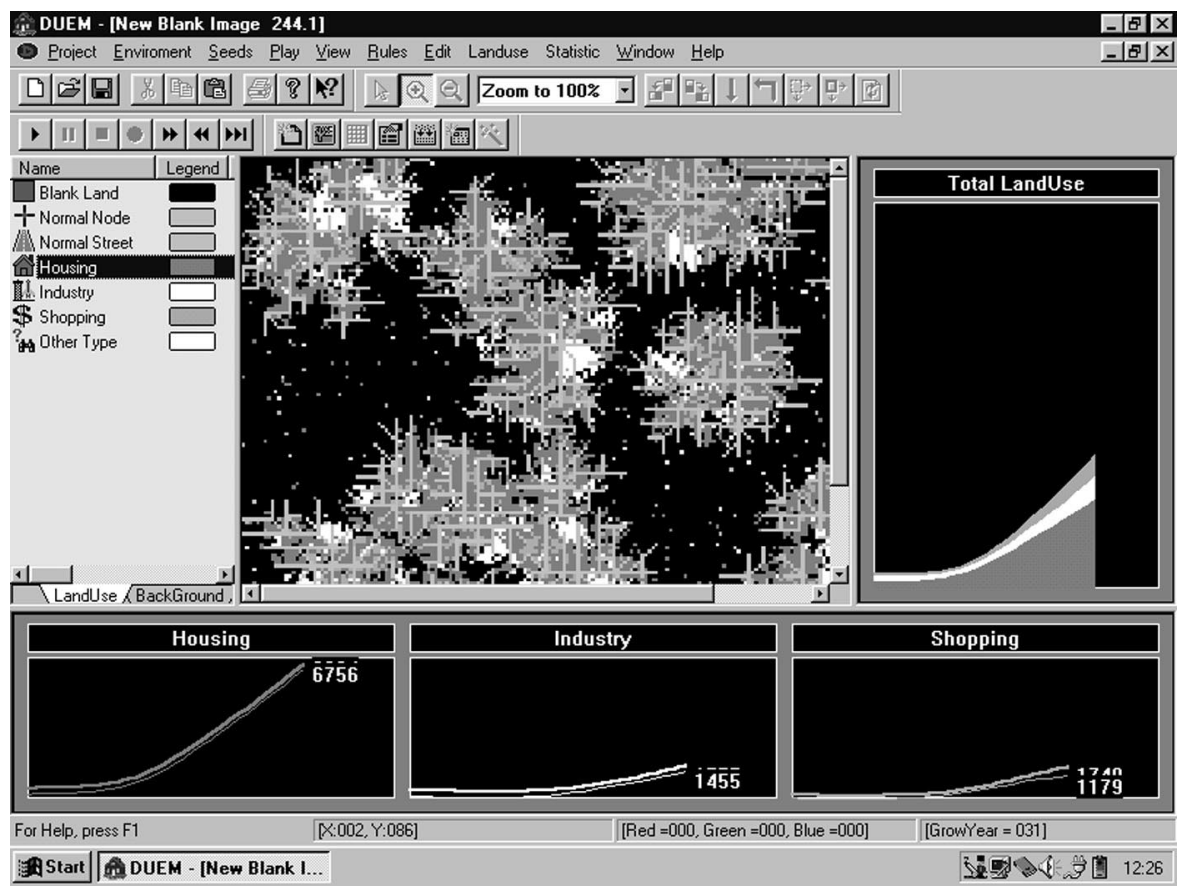

Fig. 2. The typical user interface to the spatial and temporal simulation.

as the simulation proceeds but they are also scaled to reflect movement through time and increasing volumes of activity, thus always providing a picture of the long-term dynamics of the model. The last window is simply the legend for the five land useshousing, industry, commerce/shopping, street nodes and street lengths, and vacant land. In fact, there is a sixth dummy land use which can be defined by the user, but all this indicates is that the system is potentially expandable to deal with many more land uses than those defined here.

The desktop interface consists of a main menu set at the top of the screen and four tools bars below this that contain functions that can also be accessed through the drop down menus associated with the top menu set. The menu set is shown in Fig. 3 with the drop down menus which are currently active. Many of these functions are self explanatory for they are standard to PC (and some Macintosh) interfaces, but to provide a sense of what the software contains, we will discuss these in turn. First the Project item is the equivalent of 'File' in conventional software. This enables the user to launch a new project or application or load an existing one, or to close, save or print the current application from an active window. More than one project can be launched in one session and in fact, if the projects are small enough in terms of spatial scale, then they will run simultaneously, as if in parallel although this is not a feature that is recommended. In fact, the application itself is defined under Environment which enables users to create a space with dimensions of up to $3000 \times \overline{\times} 3000$ pixels, providing the cells of the grid in which development takes place. 


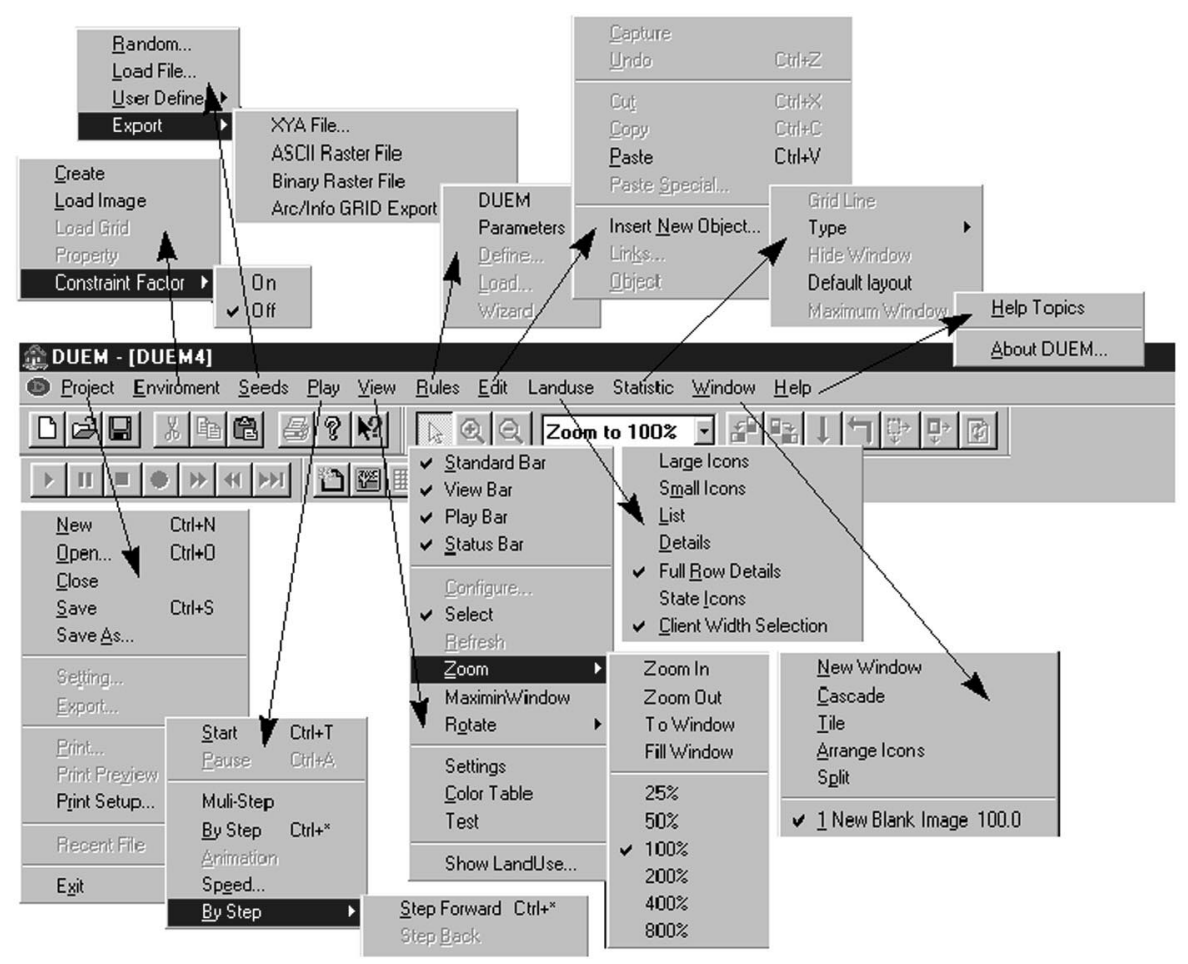

Fig. 3. The main menu set forming the graphical user interface.

At this point, it is worth noting that once this space is defined, then the user can zoom in or out, each zoom enlarging or contracting the space on the screen by $25 \%$, with the range of these zooms being $11 \times$ downward (or zoom in), and $19 \times$ upward (or zoom out). The magnifying icons on the toolbar enable this or it can be done from a menu item under View. In fact, when the software is loaded, the graph windows and the legend are set up immediately and once the spatial system window has been fixed, it is possible to resize these to give some customization to the presentation. For example, if the application only deals with one land use, say housing which might be considered to be generalized urban development, then windows can simply be suppressed leaving only the total activity graph and the spatial window which contain all the information that is needed. Under Environment, the user can also load an image as a backcloth (from various standard formats) which can be used instead of the blank sheet produced by selecting Create. This image might contain constraints on development which can be activated, if required, by clicking on the Constraint item within the drop-down menu.

When the various windows have been fixed, the user must then decide how the application is to be run. In fact at this point, the various rules defined in the previous section must be specified but will postpone discussion of this through the software until later. The item Seeds deals with the way the model runs get started. In the 
case of a hypothetical CA, it is possible to define seeds randomly by specifying the density of vacant sites and the proportions of housing, industry or commerce as well as the density of street nodes, which is what we show in Fig. 2, This routine simply locates these seeds randomly within the space. Once the model is started, a fairly large proportion may not take off or may die because the densities of relevant land uses in their neighborhoods and fields do not meet the thresholds required by the various rules which determine development. The user can also define seeds directly on the screen or load a file of seeds which can be created either in the software and exported, or created externally. At this point the user is ready to run the model, and this is activated in Play which contains the item Start. The simulation then begins; the user can stop or pause from this menu or can set the simulation to proceed one step at a time or in a multi-steps (10 time periods at once). These options are also included in the animation tool bar which is the first in the second row of tool bars in Figs. 2 and 3. One very nice feature of the software is its graphical and numerical flexibility; the user can move toolbars and windows on the screen, resizing windows, switching graphs and land use patterns on or off within these windows, and zooming in or out as the simulation proceeds. If the simulation is stopped, it can be restarted after the user has intervened in the process by adding new seeds, deleting development and/or changing the many rules that govern the way the city develops.

The other main menu items deal with ways of viewing the various decisions which have already been made. The View item enables or disables any of the four tool bars the Standard bar which activates save, print and so on, the View bar which puts zoom, rotate, and scale functions into icons, the Play (animation) bar, and the Status bar which enables the Environment and Seeds to be specified directly. It also provides the zoom and rotate options as menu items and it lets the user change the background color (Settings) and the colors of the various land uses (Color Table). The Rules item enables general parameters relating to distance, direction, density and transition (already defined) to be specified but as we will see, this has to be done for each land use and is best activated through the legend. The Edit function is disabled at present but will be used for drawing on the background grid image. Land Use enables the legend to be displayed in various ways and is only activated when the mouse is pointed at the legend. Finally Statistic which is only activated when the mouse is pointed at a graph enables graphs to be plotted with or without grid lines, to be hidden, to revert to default size or full screen size. It also enables the vertical scale of each graph to be set at the maximum value of the activity of that graph so far or to be scaled so that each graph is based on the maximum of any activity so far. The Window item is used to shift between any of the map windows that have been defined for different projects, making one of these active, or cascading, tiling, or splitting the windows on the full screen. Help provides information about DUEM, its authors and contact points as well as access to the full Help menu. What is displayed in the development window can also be controlled by the right mouse button where several of the menu/tool bar options pertaining to scaling can be activated by point and click. If the land use item is activated from the drop down menu which is brought up this way, it is possible to enable the window to show any combination of the four land uses once selected and then refreshed. 
The definition of parameter values for each of the land uses is achieved by clicking on the relevant caption box (which shows the color of each land use) in the legend. For Vacant Land, Normal Street (length) and Other Types, only the color and the title of the land use can be changed. In fact this indicates that these land uses are entirely passive within the simulation. For the other four land uses, a series of dialog boxes are opened when these are selected. Typically a box appears which contains items from which the distance function parameters, the directional locator function (as shown in Fig. 1), and the density thresholds on location in the activity field can be defined. The size of the neighborhood and the field associated with the land use are also determined at this stage, while the selection of the decay probability pertaining to whether the land use is in its initiating, mature or declining phases is also set through this dialog. In fact, there is another option for life cycle effects available in the model through a step function which determines if the activity is either initiating or mature over an entire part of the cycle. This is the one we have used in these simulations. However the life cycle probabilities as reflected through the parameters associated with the decay functions above in Eqs. (2)-(5) are also specified in this dialog if the step function is not used.

There are many parameters which need to be defined to reflect the particular urban system being simulated and the range of possible model types is enormous. The purpose of this paper is primarily to give readers a sense of the way complex dynamics can be embedded into GIS-oriented software to enable comprehensive and intelligible simulations to be performed. In future papers we will begin a systematic exploration of the parameter spaces that can be set up through this software. In the next section, we will illustrate five typical examples which although very different, barely cover the range of possibilities which this simulator can portray. But these do give some sense in which various model classes can be represented and we will conclude this section with a brief discussion of these model types. What is not possible within the software is the simulation of highly regular symmetric CA-like urban forms, due to the fact that although development can occur in regular neighborhoods and fields, symmetry is always broken through the selection of a single unit of land use in each field at each time. If development has the same probability of occurring in any regular field around a site, then a choice has to be made to locate this development at one site only. This breaks any symmetry that might be present in the model, but it is also characteristic of how real world development takes place.

The biggest variations that can be made in these models pertain to the dimensions of the spatial system, the number of land uses that can be handled and the way development relates to its neighborhood and field. As we have already noted, we can specify the same system at very different scales (in terms of the number of development cells), or different sized systems at the same scale. We can thus use the software to simulate single cities or systems of cities although as we change scale in this way, the manner in which land uses become significant also changes. Much will depend upon the particular application but in the next section we will look at single city and multiple city examples which illustrate these differences. We can also define examples which are based on single or multiple land uses. Many CA models have concentrated in the past on the simple distinction between urban and non-urban 
development, simulating the transition from rural to urban for example. We can easily represent such examples in this software and we will do so in the next section for one of the best ways to use the simulator, is to develop simple examples and watch them develop. However, another important issue reflects the size of the field and the neighborhood used by different land uses. If we constrain these neighborhoods and fields, then we generate compact development. If we define very large fields, we can generate quite dispersed urban forms but at the same time, we may define problems in which development dies out because neighborhoods and fields are not well-defined in terms of their respective sizes to one other.

These are issues yet to be explored but we will introduce the difference between compact and dispersed forms later. Moreover, we can define quite different life cycle effects in which development can interact temporally with the size of the spatial system. For example, if we define a small system of say $50 \times 50$ cells, and if development is in its initiating phase for say 30 years and then in its mature or inactive phase for another 30 years, then development will die out as the activity reaches the edge of the system and passes into its mature phase. In short, the temporal cycle interacts with the spatial cycle in ways that must be anticipated and controlled for. Finally, there is some difficulty in allocating streets to entire grid cells which is clearly a very crude way of representation. This is a problem that will be tackled in future developments as it is possible to let cells share land uses. However the attraction of the CA-like logic is that each cell has only one state, in this case land use. In the future, it is our intention to relax this stricture for transport routes and to open windows to display different types and combinations of the various uses.

\section{Simulating generically different urban forms}

There are many parameter values to be set within this model which will lead to very different spatial simulations and a thorough exploration and understanding of the model must be based on charting the solution space of possibilities which different combinations of these values generate. This is the time honored way of exploring complexity of this kind as used in other such models by the authors (Batty \& Longley, 1994; Batty \& Xie, 1997) but before we attempt this, there is a general set of explorations that we need to undertake. Different parameter values will generate different forms but considering the model in perspective, it is clear that we need some general testing of its basic properties before such detailed repetitive tests are initiated. These are presented in this paper; first in terms of the model's dynamics and temporal scaling; second in terms of limits on the capacity of the system and the transition from new to mature regimes; third in terms of the way the model can be adapted to deal with different types and combinations of activities ranging from general urban development to specific land uses; fourth in terms of how the model can generate different effects at different spatial scales, in particular simulating systems of cities or structures internal to cities themselves; and finally in terms of how the model generates different densities of development from compact to highly decentralized. We will develop these through a series of examples. 
In fact, all the examples are based either on the default values of parameters which we have preset in the software or on slight variations of these. These values are given in Table 1 and some explanation of what they generate is in order. For each land use, the neighborhood sizes are based on the $3 \times 3$ Moore neighborhood which nest within $5 \times 5$ fields with the exception of the street development which is embedded in a much large field size of $8 \times 8$. The distance decay functions are all linear with a decay rate of 0.8 meaning that the probability of land uses spawning other uses in the field decays by $80 \%$ per unit pixel. This is strong effect and as such it implies that the default model will generate highly compact growth. There are no distorting effects in terms of direction, that is, the probabilities of development are symmetric around the neighborhood cell in question. In terms of housing, then there must be at least $80 \%$ housing in the neighborhood for new housing to be spawned but there need only be 15 and $25 \%$ of industry and shopping, respectively, for the new use to be either of these two. For industry, the probabilities of the new use being housing or shopping are $40 \%$ whereas for shopping the probabilities of the new use being housing or industry are $20 \%$ respectively. In short, industry and shopping do not spawn more industry or shopping per se although we are conscious that these rules are fairly arbitrary and will be subject to much more scrutiny when realistic applications are attempted. In terms of the minimum density in each neighborhood for streets to be developed, these are 10, 60 and 70\% for housing, industry and shopping, respectively. If a less compact city is to be generated, then these proportions should be relaxed.

The values in Table 1 were used to generate the simulation shown in Fig. 2. In that example, the simulation began with randomly placed seeds for each land use on a grid of dimension $150 \times 200$. This generates a set of small villages which begin to grow according to the parameters set in Table 1. This application provides an excellent example of the ability of the model to generate clear ideas about urban dynamics. Fig. 2 shows how from a random seeding involving 200 housing, 70 shopping and 100 industrial sites with 50 street nodes, 12 settlements are generated.

Table 1

Default parameter values for the basic model runs

\begin{tabular}{lllll}
\hline Parameter & Housing & Industry & Shopping & Street nodes \\
\hline Distance decay & Linear & Linear & Linear & Linear \\
& $(\mathrm{Q}=1 \alpha=0.8)$ & $(\mathrm{Q}=1 \alpha=0.8)$ & $(\mathrm{Q}=1 \alpha=0.8)$ & $(\mathrm{Q}=1 \alpha=0.8)$ \\
Direction & Symmetric & Symmetric & Symmetric & Symmetric \\
Neighborhood size $\Omega_{1}$ & $3 \times 3$ & $3 \times 3$ & $3 \times 3$ & $3 \times 3$ \\
Field size $\Omega_{2}$ & $5 \times 5$ & $5 \times 5$ & $5 \times 5$ & $8 \times 8$ \\
Minimum street density & $5 \%$ & $15 \%$ & - & - \\
Maximum housing density & $80 \%$ & $40 \%$ & $20 \%$ & $10 \%$ \\
Maximum industry density & $15 \%$ & - & $20 \%$ & $60 \%$ \\
Maximum shopping density & $25 \%$ & $40 \%$ & - & $70 \%$ \\
Initiating growth period & 30 & 15 & 10 & - \\
Mature growth period & 30 & 30 & 30 & - \\
Quiescent (dying) period & 0 & 0 & 0 & - \\
\hline
\end{tabular}


Many of the random seeds never kick in as they do not meet the required threshold conditions for street and related growth. However, the picture in Fig. 2 after 30 time periods is one of exponential growth where all activity is increasing.

In fact as the simulation continues, the space begins to fill up and as it does so, growth is inevitably constrained. Fig. 4 at time period 150 shows how the activities peak, and then decline a little only to release more space as they progress from their initiating to mature periods and thence die off, making room for new growth. These cycles continue indefinitely but as the simulation proceeds, then what happens is that some form of steady state emerges in which the proportion of activities begins to change and stabilise. In fact, we are not sure that there is long-term equilibrium in these terms and this is something to be explored in the future. But Fig. 5, which is a record of progress at time period 377, shows that housing gradually declines as a proportion of total activity and then seemingly stabilizes while shopping grows and then declines a little with industry growing at first but thence declining and then beginning to grow again at the end of the period shown. In this sense, the software provides an excellent diagnostic for exploring the effect of different parameter values on the model's dynamics.

Our second foray into model dynamics involves developing a model based on a single city in a fixed space. This leads to logistic growth of the kind seen in Figs. 4 and 5 but this time the emphasis is upon the time during which the growth phase is active and the space over which this takes place. We will choose just one activity-

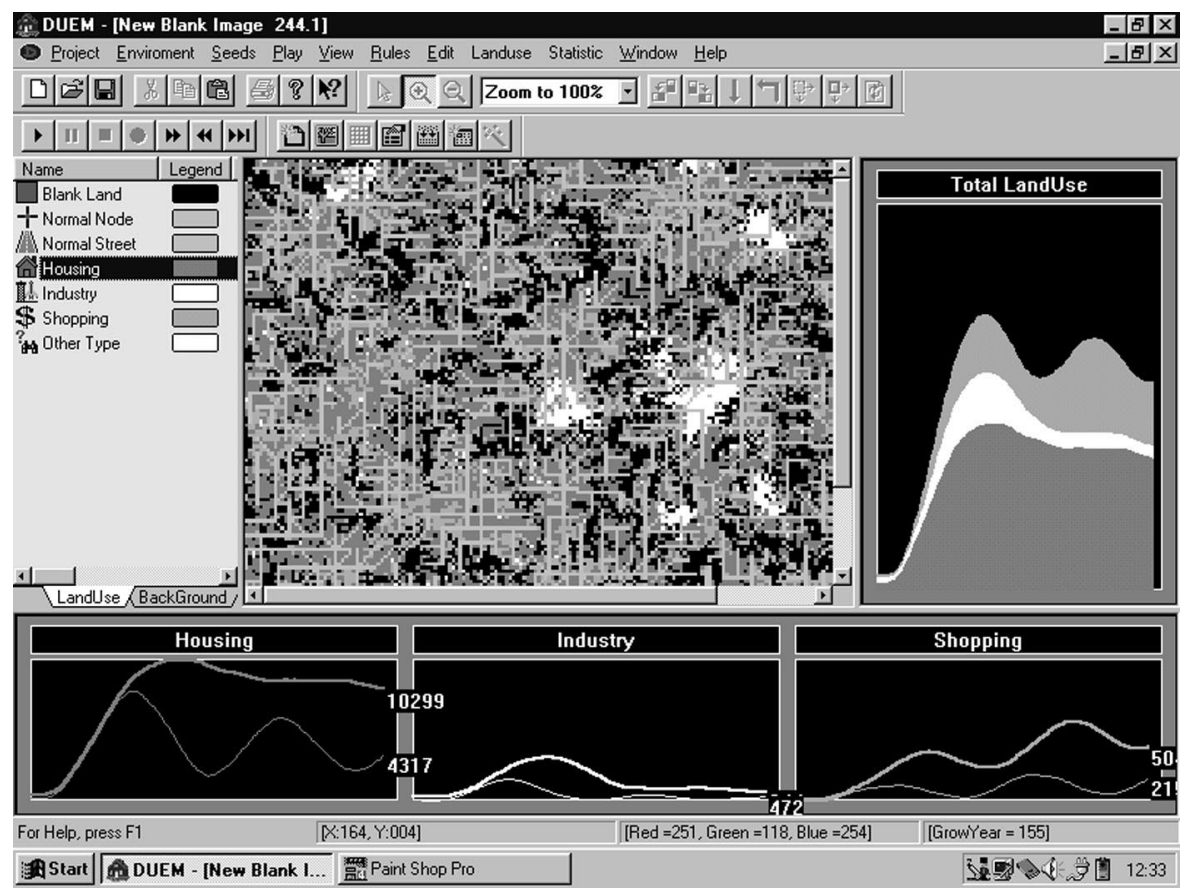

Fig. 4. Short-term logistic dynamics for a system of cities. 


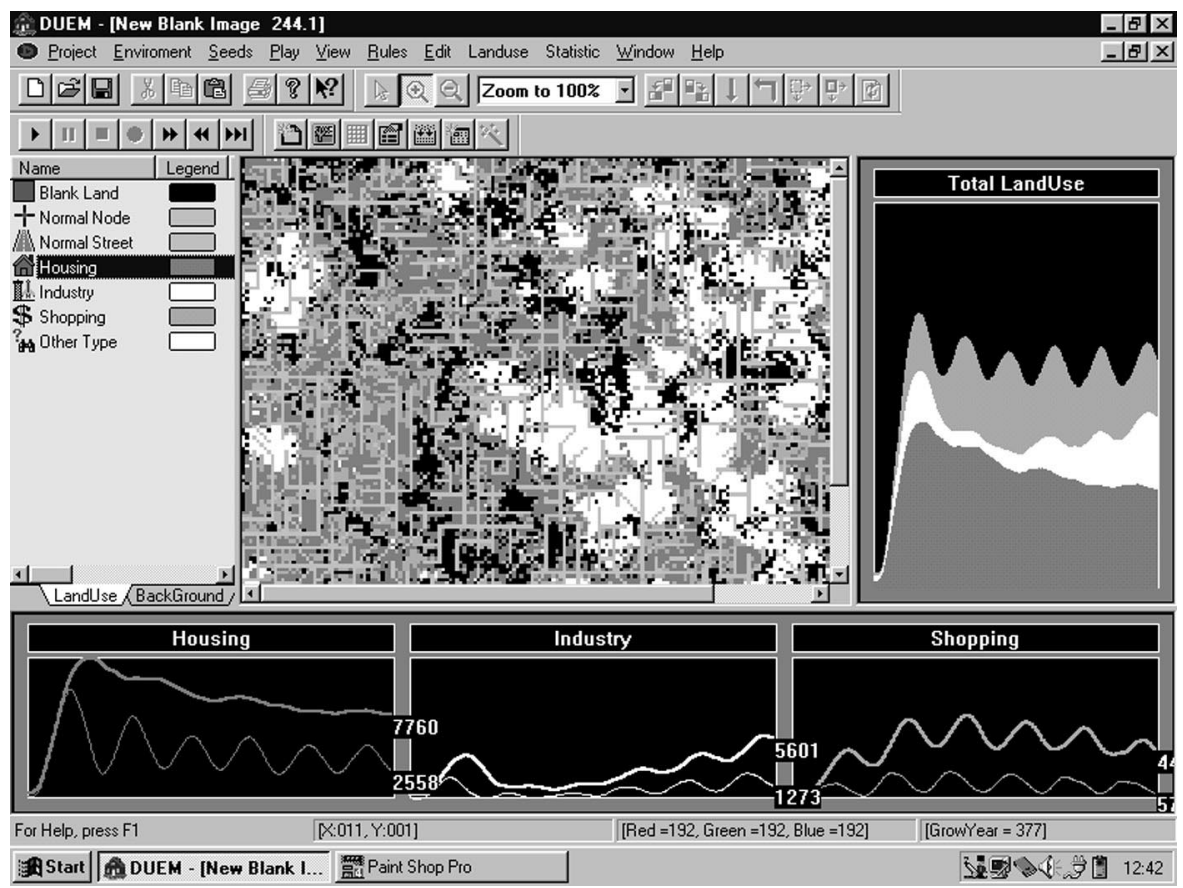

Fig. 5. Longer-term cycles of growth and decline.

urban development say - and thus the model will provide a demonstration of how urban development diffuses around a central city site. But as new development only actively spawns further development for a fixed initiating period, then this demonstrates a process akin to the invasion and succession of land use in large cities whereby new growth stops generating further growth, eventually dying and thence providing room for further new growth. In effect, what we will show here is that if the period of active growth is too long and the size of the spatial system too small, then growth will simply die out because no land is made available for further growth in the vicinity of existing development. In short, what is happening is that the time cycle of the system is co-ordinated with the spatial location: as growth moves out from the centre, this is related to the distance from the centre and development occurs in waves which are a function of the way the growth phase coincides with location. The neighborhood size and growth phase can thus be chosen so that the waves of growth simply passes through the system and fails to activate new growth before it dies.

In Fig. 6, we show an example of this 'wave of development effect' which is akin to a pulsating clock generating successive waves of growth about a central point from a single seed. In this case, the system is set up as $100 \times 100$ pixels, the growth phase is 10 time periods, the mature phase also 10, no streets are required to provide a threshold for development and there is no transition from housing (which is classed as generic urban development') to other uses. In effect, a wave of growth develops 
and spreads fairly evenly around a central point. When the earliest development ages to 10, it does not spawn new growth and when it reaches age 20 it dies and makes land available for new growth. The waves of growth are thus around 20 units of distance-read time-wide, and these move slowly across the space in ever widening rings. In fact, the symmetry of the original seed is quickly broken and although, by and large, the circularity remains, several cores develop and dissolve as randomness of locational choice distorts the original pattern. Moreover as Fig. 6 shows, these waves are also apparent from the total volumes of activity generated which rise and fall with the ebb and flow of development.

Our third experiment involves generating dispersed settlement patterns. The default parameter values presented in Table 1 suggest very compact forms and it is easy to see how dispersion can be introduced. This involves defining much larger fields and neighborhoods for each land use and flattening the distance functions which are used to compute the probabilities of location with respect to land uses located at the centre of each neighborhood. In Fig. 7, we first show a compact growth around a central seed for urban development in general using the default parameter values of the previous experiment but also ensuring that all activity is initiating and that it never passes to its mature phase. We then relax these, widening the field from $5 \times 5$ to $25 \times 25$, the neighborhood from $3 \times 3$ to $10 \times 10$, and setting the slope of the linear distance function equal to 0.1 . This generates a more dispersed pattern but it is clear that true dispersion does not depend upon all land being available for development. We can generate an even more dispersed pattern by ensuring that land is cycled through its initiating and mature stages immediately and this enables a much large quantity of land to remain and to become vacant during
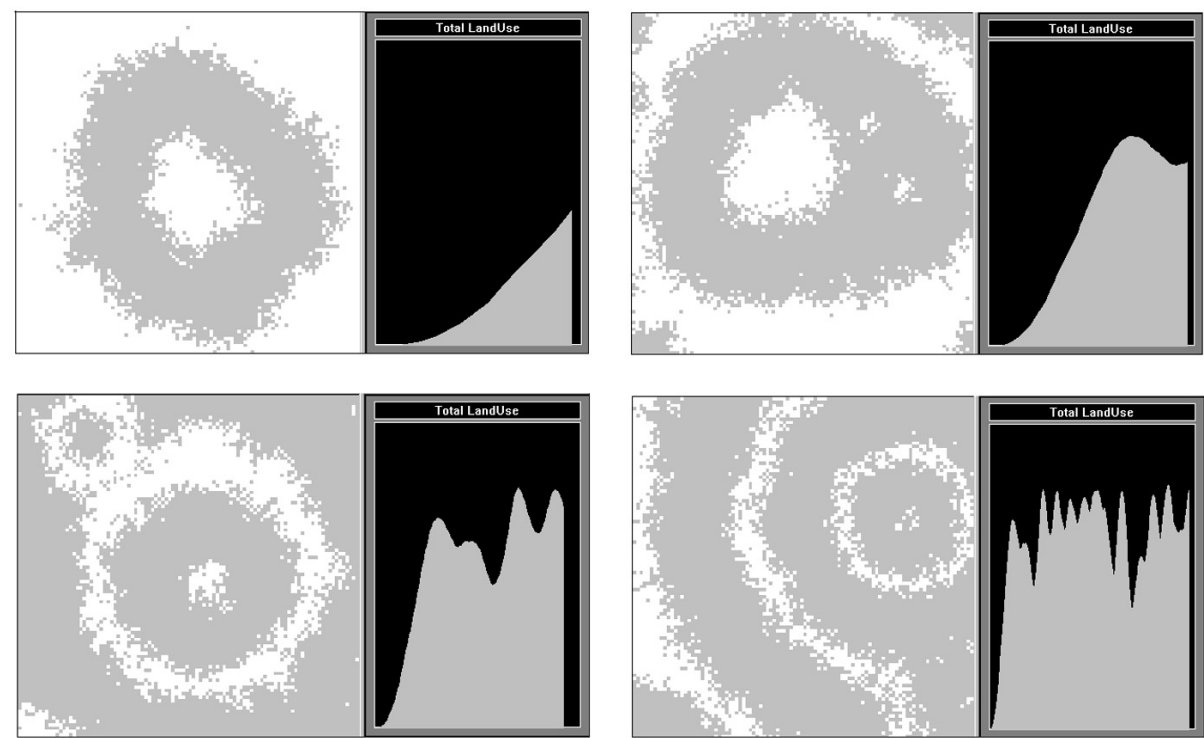

Fig. 6. Waves of urban growth reflecting cycles of maturing development. 

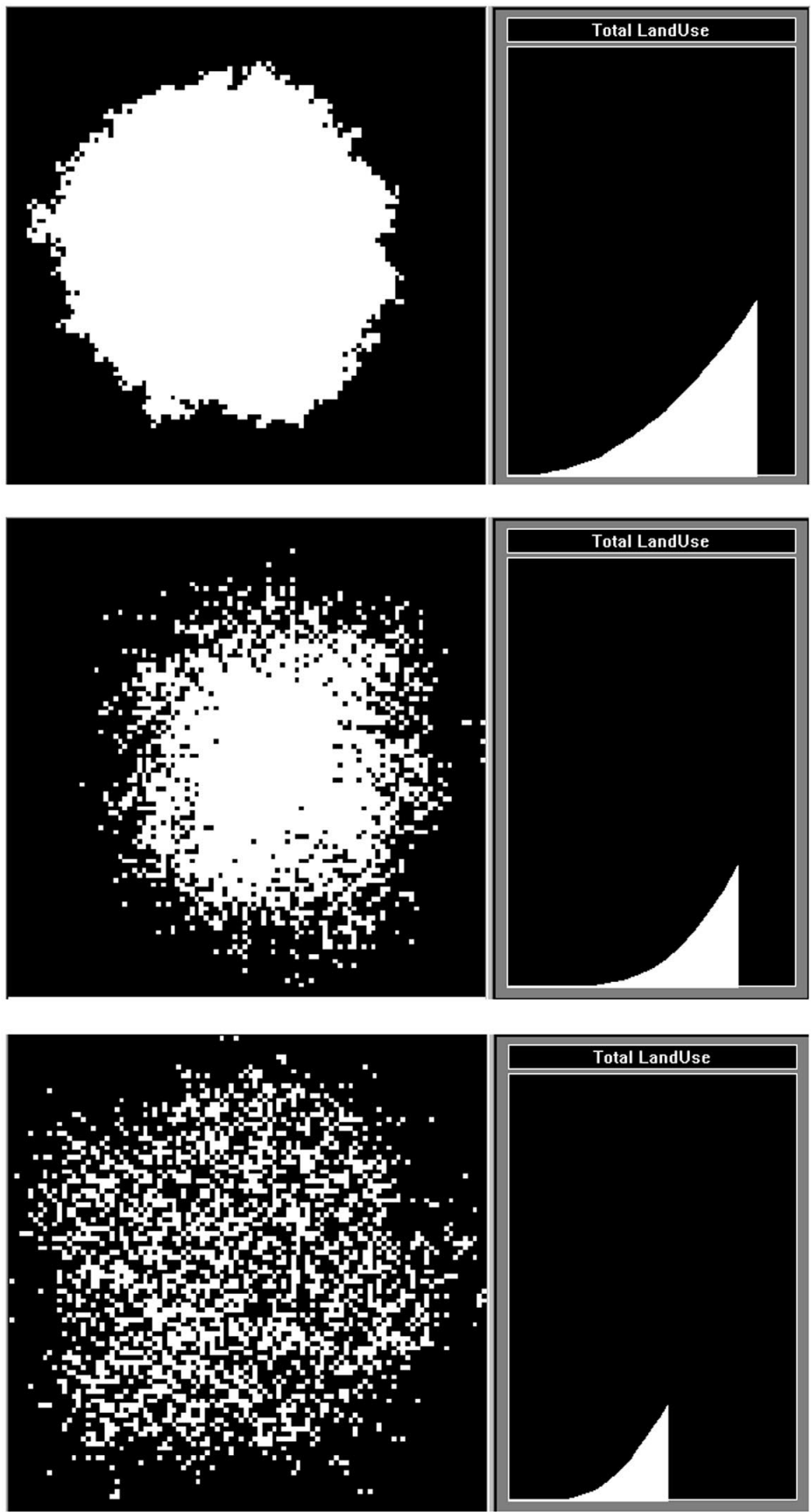

Fig. 7. Compact to dispersed urban growth. 
the development process thus generating a much more emphatic pattern of dispersion as shown in the last example in Fig. 7. How these patterns are reinforced or countered when several land uses are made interdependent, as in the complete model, will be a matter for further research which we will report in a later paper.

Our fourth demonstration of the software both changes the scale of application from free-standing towns to systems of cities and also introduces the idea that there is some minimum threshold of activity necessary in a region before towns can begin to grow. Our software is able to generate many random configurations of land uses which are the building blocks of urban development from which towns may or may not grow. In a space which measures $257 \times 350$ pixels (the default setting of the software), we can randomly locate these building blocks with different proportions of vacant, housing, industrial, shopping and street land uses. In fact, with anything more than $97 \%$ of the land set as vacant no towns will develop. Below this, with $95 \%$ set as vacant, $2 \%$ as housing, $2 \%$ as industry and $1 \%$ as shopping with some judiciously placed street nodes and lengths, urban development takes off. If you like, this is the 'origin of urban life' which occurs when the building blocks of urban development are mixed together randomly in this soup. With these proportions, around 8 clusters, while if the proportion of vacant land is reduced to $90 \%$ and the proportion of housing raised to $4 \%$, industry and shopping $3 \%$, then up to 25 clusters emerge. As the proportion of vacant land falls, less clusters appear as the proportion of street nodes is constant in our experiments but this does illustrate that the model is able to generate spontaneous growth from random seeding. Moreover, it is also clear that the size of the clusters grown is consistent with rank-size relations due to the fact that the random positioning of the building blocks generates such relations in the first instance and this preconditions further urban growth.

We have generated a system of cities through 240 time periods using a soup based on $90 \%$ vacant land, with $4 \%$ housing, and just under $3 \%$ industry and shopping with a fraction of cells being given over to streets. In Fig. 8, we show the evolution of this system at time periods 30,60, 120 and 240, where we illustrate housing development separately from street development. The streets remain fixed of course in this model which mirrors the inertia of such infrastructures in real cities, although it is clear that housing is subject to the initiation and maturing processes which are central to the operation of the model. Due to limits on space, we are not able to show industrial and shopping land uses or the system trajectories associated with this growth. Nevertheless, this does show how the system builds up and is reminiscent of the way towns fuse together to form metropolitan regions of the kind which are characteristic of Western Europe and North America. As yet we have not attempted to simulate edge city effects in these models but this is an important issue for future research.

Our last example changes the scale yet again. In CA models, we should be able to simulate much more local scale development of the kind relevant to the development of housing estates and other land parcels. In Fig. 9, we show a simple rural route pattern with some housing developed in ribbon development form. Using the default rules, we have grown this system through 50 time periods and it is clear that a town of sorts is beginning to grow from the original seeds. In fact, what happens is that 

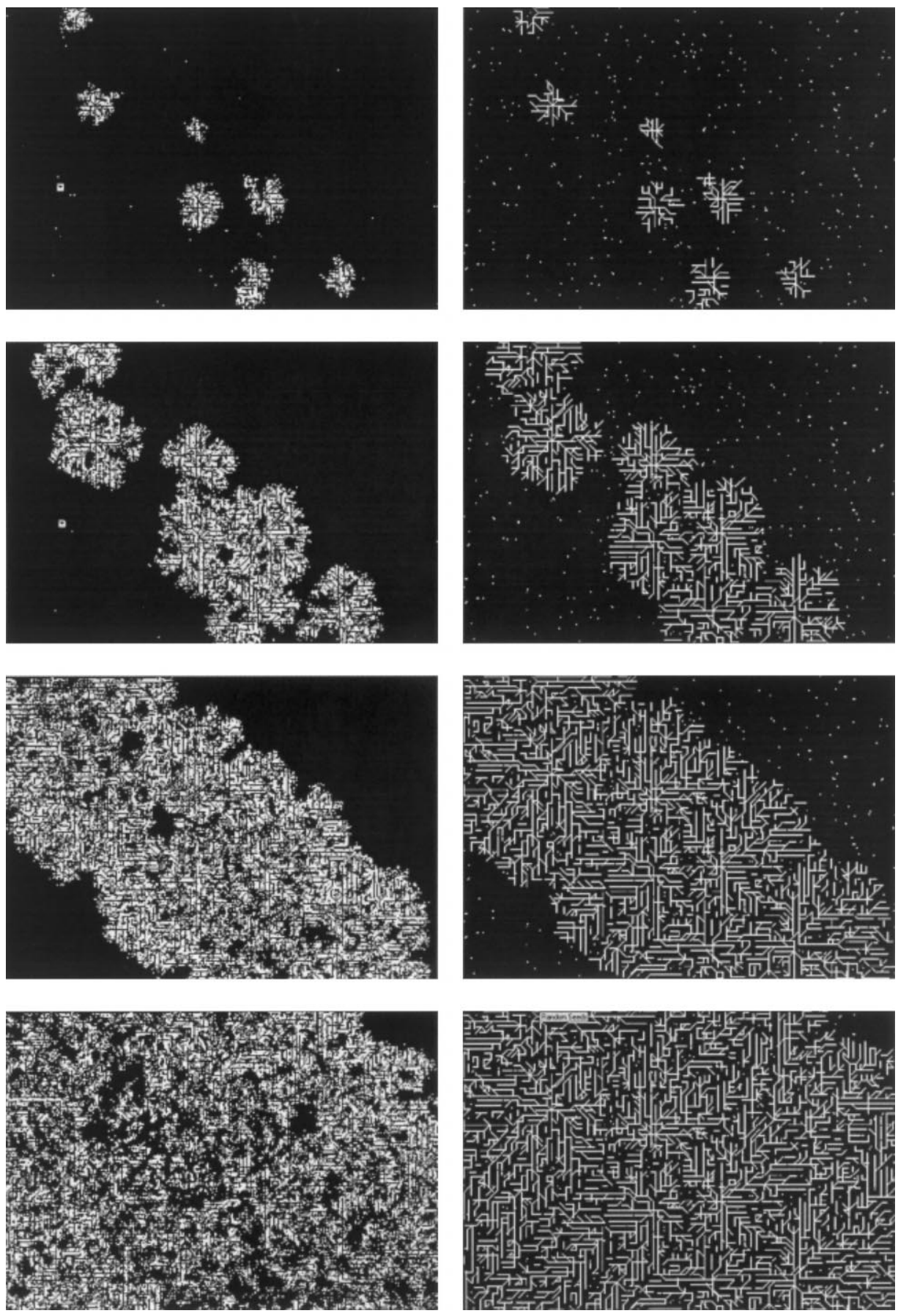

Fig. 8. Housing and street development in system of fusing cities. 


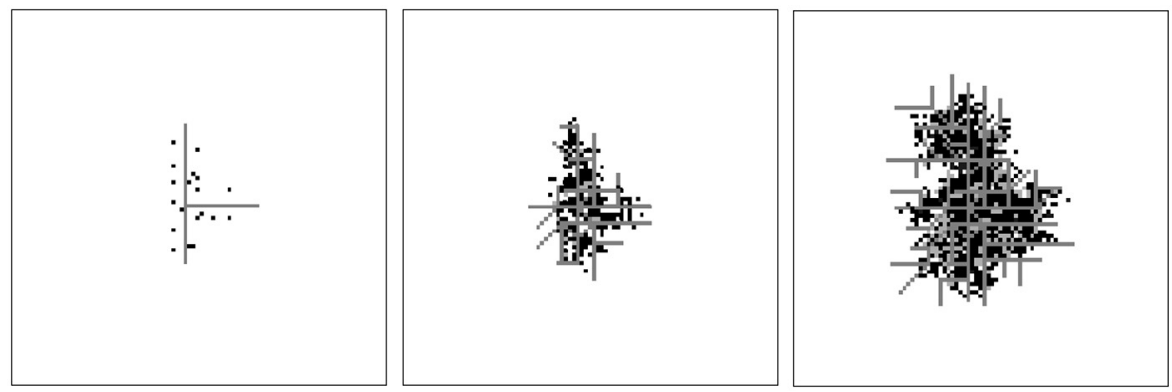

Fig. 9. Village and town from dispersed rural settlement.

the linear form of the original growth gradually becomes radial for the rules of development embedded in the model favor circularity. If we let this growth continue then the town becomes ever more circular. In further research what we plan to do is to find the rule sets that generate different types of town form from simple lowdensity linear forms such as the one used to seed this growth, in the belief that the model is sufficiently general to enable a wide array of urban development to be simulated across many different scales and through many different numbers of time periods. We have not demonstrated this in any definitive way here although we have given some idea of what is possible.

\section{Future developments: software and applications}

A systematic exploration of the model is already underway, concentrating on the inter-relationships between model parameters and the way different values lead to generically different forms. We need to be in the position where we are able to state with confidence the range of parameter values that will lead to different forms so that we can then begin to fine tune the model to simulate urban structures at very different scales and over different time periods. In short, we are embarking on an exploration of the multi-dimensional parameter space in the manner that we have suggested in previous papers (Batty and Xie, 1997).

We are also developing new ways of calibrating these types of model so that the parameters might be chosen to optimize the goodness of fit to known urban patterns. In fact, a variety of new measures of fit need to be developed of a very different nature from those used in traditional land use - transportation modeling (Batty, 1976), and we will begin to explore these when we develop applications. There are several issues that remain unresolved in terms of the model structure, in particular the way the model is able to handle growth and decline. As in this genus of models where development is generated largely in response to what is happening locally, it is difficult to control the total quantities of activity which are generated. In more practical urban models, usually the link between location and growth is severed in that growth or change is forecast separately and actual change in particular locations is scaled to meet such targets. In CA models, growth and location are 
closely dependent and although this is clearly a much more realistic assumption with respect to how cities actually develop, there has been very little emphasis on controlling total growth in these kinds of model. We need to explore the effect not only of the mechanisms which link location and growth in these models but also the way the parameter values affect activity change. This is necessary before we turn to applications.

We must also develop a better set of default parameter values and extend the graphical user interface to make it easier to specify these values. Finally we need to consider spatial interactions within such models. Unlike most CA models to date, this model does treat route structures - streets - as integral to the development of land use but it is possible to go one step further and to consider how relationships between activities can be modeled in terms of interactions and flows. In this sense, the model can be extended to deal with the development of network structures integrated with spatial stocks and flows which form the activity dynamics of the urban system.

The model can be adapted to simulate urban development over very different time periods but in our first applications, we will concentrate on using the model to simulate marginal changes over decanal periods. Following the tradition of diffusive simulation pioneered by Hagerstrand $(1953,1967)$ and developed for modeling residential growth (Chapin \& Weiss, 1968; Batty \& Xie, 1994a), we are currently developing the model to simulate change between 1980 and 1990 in the town of Ann Arbor, MI, which is in the western sector of the Detroit region. There is excellent data for this region from early simulations by Tobler (1970) to the current digital base for Wayne County which is available at parcel-based level for 1970, 1980 and 1990. We are developing a simulation of urban change between these two dates using change in the 1970 to 1980 base as seeds for the 1980 to 1990 change. In this application, we will develop new ways of calibrating these models based on such data sets, reporting these developments and the way they will influence and change the model design in future papers.

\section{Acknowledgements}

This project is part funded by the US National Science Foundation (NSF Grant 9752086) and by the UK Office of Science and Technology Foresight Challenge (EPSRC GR/L54950).

\section{References}

Allen, P. M. (1998). Cities and regions as self-organizing systems: models of complexity. New York: Gordon and Breach.

Batty, M. (1971). Modelling cities as dynamic systems. Nature, 231, 425-428.

Batty, M. (1976). Urban modelling: algorithms, calibrations, predictions. Cambridge, UK: Cambridge University Press.

Batty, M. (1991). Cities as fractals: simulating growth and form. In T. Crilly, R. A. Earnshaw, \& H. Jones, Fractals and chaos (pp. 41-69). New York: Springer-Verlag. 
Batty, M., \& Longley, P. A. (1994). Fractal cities: a geometry of form and function. San Diego, CA: Academic Press.

Batty, M., \& Xie, Y. (1994a). From cells to cities. Environment and Planning B, 21, 31-48.

Batty, M., \& Xie, Y. (1994b). Urban analysis in a GIS environment: population density modeling using ARC/INFO. In A. S. Fotheringham, \& P. A. Rogerson, Spatial analysis and GIS (pp. 189-219). London: Taylor and Francis.

Batty, M., \& Xie, Y. (1997). Possible urban automata. Environment and Planning B, 24, 175-192.

Burrough, P. A. (1988). Dynamic modeling and geocomputation. In P. A. Longley, S. M. Brookes, R. McDonnell, \& B. MacMillan, Geocomputation: a primer (pp. 165-191). Chichester, UK: Wiley.

Chapin, F. S., \& Weiss, S. F. (1968). A probabilistic model for residential growth. Transportation Research, 2, 375-390.

Clarke, K. C., Hoppen, S., \& Gaydos, L. J. (1997). A self-modifying cellular automaton model of historical urbanization in the San Francisco Bay area. Environment and Planning B, 24, 247-261.

Clarke, K. C., \& Gaydos, L. J. (1998). Loose-coupling of a cellular automaton model and GIS: long-term growth prediction for the San Francisco and Washington/Baltimore. International Journal of Geographical Information Science, 12, 699-714.

Couclelis, H. (1997). From cellular automata models to urban models: new principles for model development and implementation. Environment and Planning B, 24, 165-174.

Densham, P. J. (1996). Visual interactive locational analysis. In P. Longley, \& M. Batty, Spatial analysis: modelling in a GIS environment (pp. 185-205). Cambridge, UK: GeoInformation International.

Forrester, J. W. (1969). Urban dynamics. Cambridge, MA: MIT Press.

Hagerstrand, T. A. (1953, 1967). Innovation diffusion as a spatial process. Chicago, IL: University of Chicago Press.

Itami, R. M. (1994). Simulating spatial dynamics: cellular automata theory. Landscape and Urban Policy, $30,27-47$.

Makse, H. A., Havlin, S., \& Stanley, G. (1995). Modeling urban growth patterns. Nature, 377, 608-612.

Makse, H. A., Andrade, J. S., Jr., Batty, M., Havlin, S., \& Stanley, H. E. (1998). Modeling urban growth patterns with correlated percolation. Physical Review E, 58(6), 7054-7062.

Meaille, R., \& Waled, L. (1990). Using geographical information systems and satellite imagery within a numerical simulation of regional urban growth. International Journal of Geographical Information Systems, 4, 445-456.

Mikula, B., Mathian, H., Pumain, D., \& Sanders, L. (1996). Integrating dynamic spatial models with GIS. In P. Longley, \& M. Batty, Spatial analysis: modelling in a GIS environment (pp. 283-295). Cambridge, UK: GeoInformation International.

Rinaldi, E. (1998) AUGH!: generalized urban automata with help!, Users Guide 1.8 Version, DAEST, Collana Statema No. 2, Instituto Universitario Di Archittetura Venezia, Venice, Italy.

Tamayo, P., \& Hartman, H. (1989). Cellular automata, reaction-diffusion systems, and the origin of life. In C. G. Langton, Artificial life (pp. 105-124). Reading, MA: Addison-Wesley.

Tobler, W. R. (1970). A computer movie simulating population growth in the Detroit region. Economic Geography, 42, 234-240.

Toffoli, T., \& Margolus, N. (1987). Cellular automata machines: a new environment for modeling. Cambridge, MA: MIT Press.

Wagner, D. F. (1997). Cellular automata and geographic information systems. Environment and Planning $B, 24,219-234$.

Wegener, M. (1994). Operational urban models: state of the art. Journal of the American Planning Association, 60(1), 17-29.

White, R. W., \& Engelen, G. (1993). Cellular automata and fractal urban form: a cellular modelling approach to the evolution of urban land use patterns. Environment and Planning A, 25, 1175-1193.

Wu, F. (1998). SimLand: a prototype to simulate land conversion through the integrated GIS and CA with AHP-derived transition rules. International Journal of Geographical Information Science, 12, 63-82.

Xie, Y. (1994). Analytical models and algorithms for cellular urban dynamics. PhD dissertation, State University of New York at Buffalo, Buffalo, NY.

Xie, Y. (1996). A generalized model for cellular urban dynamics. Geographical Analysis, 28, 350-373. 
Yeh, A. G., \& Li, X. (1998). Modelling sustainable urban forms using extended cellular automata and GIS. In T. K. Poiker, \& N. Chrisman, Proceedings of the 8th international symposium on spatial data handling. International Geographic Union, 11-15 July, Vancouver, Canada, pp. 453-464. 